\title{
Energetics of interlayer binding in graphite: The semiempirical approach revisited
}

\author{
Masayuki Hasegawa, ${ }^{1, *}$ Kazume Nishidate, ${ }^{2}$ and Hiroshi Iyetomi ${ }^{3}$ \\ ${ }^{1}$ Department of Materials Science and Engineering, Iwate University, Morioka 020-8551, Japan \\ ${ }^{2}$ Department of Electrical and Electronic Engineering, Iwate University, Morioka 020-8551, Japan \\ ${ }^{3}$ Department of Physics, Niigata University, Niigata 950-2181, Japan
}

(Received 10 May 2007; published 18 September 2007)

\begin{abstract}
We have developed a semiempirical method to obtain interlayer binding energy of graphite in the previous work [M. Hasegawa and K. Nishidate, Phys. Rev. B 70, 205431 (2004)]. In the present paper, we revisit this approach and develop an improved method, in which $a b$ initio calculations based on the density functional theory (DFT) are also corrected through an empirical atom-atom van der Waals (vdW) interaction. The local density approximation (LDA) and generalized gradient approximation (GGA) are used in the DFT calculations. The parametrized damping function introduced to modify the asymptotic atom-atom vdW interaction is more flexible than the previous ones and covers a wider range of possibility in correcting for the approximate DFT calculations. The damping function is determined empirically by imposing the condition that the experimental interlayer spacing, in-plane lattice constant, and $c$-axis elastic constant are reproduced. We also require consistency between the LDA- and GGA-based methods (LDA+vdW, GGA+vdW) as the theoretically motivated necessary condition. The interlayer binding energy obtained by this method is $60.4 \mathrm{meV} /$ atom at $T=0 \mathrm{~K}$. The result of $\sim 54 \mathrm{meV} /$ atom at room temperature corrected by the thermal effect is consistent with the most recent experiment, $52 \pm 5$ eV/atom [R. Zacharia et al., Phys. Rev. B 69, 155406 (2004)]. The atom-atom vdW interaction obtained by the present semiempirical method favorably corrects for the overbinding and underbinding nature of the LDA and GGA, respectively, in the in-plane energetics of graphite. That interaction also provides a useful starting point for the studies of energetics of other graphitic systems such as fullerenes and carbon nanotubes.
\end{abstract}

DOI: 10.1103/PhysRevB.76.115424

PACS number(s): 61.50.Lt, 71.15.Mb, 71.15.Nc

\section{INTRODUCTION}

Graphite is a typical solid of layered structure and is characterized by a relatively weak interlayer binding compared to a rather strong binding within the layers. The covalent bonding due to the threefold $s p^{2}$ hybridized orbital is responsible for the strong binding within the layers, and the resulting hexagonal network of carbon atoms provides a useful starting point in the studies of other graphitic systems such as fullerenes and carbon nanotubes. ${ }^{1}$ The weak interlayer binding is supposed to arise from the dispersion or van der Waals $(\mathrm{vdW})$ interaction and the overlap of partially occupied $2 p_{z}$ orbitals perpendicular to the graphitic planes. In general, the $\mathrm{vdW}$ interaction has only a minor effect on the bulk properties of dense and hard matter but plays a crucial role for the structure and dynamics of systems with sparse electron distribution. Binding energetics in graphitic structures is one of such typical examples and has been attracting much theoretical attention as a basic issue. ${ }^{2}$

Three types of theoretical methods have been used to investigate the energetics of interlayer binding in graphite. The first method is based on the model potential, mostly Lennard-Jones (LJ) potential, whose parameters are usually determined empirically. This empirical method has been successful in providing a unified, consistent description of the properties that depend on the weak interactions in graphitic systems. ${ }^{3-5}$ The second ab initio method has most often been based on the density functional theory (DFT) ${ }^{6,7}$ and in these DFT calculations, the local density approximation (LDA) ${ }^{7}$ and generalized density-gradient approximation (GGA) with various flavors (e.g., Refs. 8-13) have been used as the stan- dard approximations. Many authors have performed such calculations for graphite and found that LDA yields better results for the $c$-axis lattice constant and interlayer binding energy (i.e., exfoliation energy) than GGA. ${ }^{14-25}$ Earlier results of these calculations are summarized in Ref. 24. The $\mathrm{vdW}$ interaction or dispersive interaction may be taken into account to some extent in these local and semilocal approximations at short and intermediate distances where the overlap of electron density is appreciable, but certainly cannot be captured at large separation where the overlap is negligible. The relation between the empirical and ab initio DFT methods and the apparent success of LDA for graphitic systems have been discussed in some detail by Girifalco and Hodak. ${ }^{2}$ In an attempt among several challenges to fully incorporate $\mathrm{vdW}$ interaction in the DFT (e.g., references cited in Refs. 26 and 27), Rydberg et al. have developed a tractable vdW density functional (vdW-DF) for planar geometry ${ }^{26}$ and applied it with moderate success to graphite, boron nitride (BN), and molybdenum sulfide of layered structures. ${ }^{27,28}$ More recently, they developed an vdW-DF for general geometry and applied it with better success to the bilayer graphene sheets and adsorption energetics of organic molecules on a graphitic surface. ${ }^{29,30}$ The implementation of these vdW-DF methods requires heavy computational cost, and their accuracy is still not fully satisfactory, as we see for graphite. The theoretical and practical limitations of DFT calculations and the $a d$ hoc nature of empirical methods have led to the third method as a compromise between the two, and several attempts have been made for graphite and other systems. ${ }^{24,31-38}$ In this semiempirical method, results of ab initio DFT calculations are supplemented with an empirical atom-atom vdW interaction modified by a damping function. This modification is 
intended to correct for the $r^{-6}$ singular behavior at short distances and, though not necessarily accepted commonly, to take care of the possible situation that the $\mathrm{vdW}$ interaction at short and intermediate distances is already taken into account to some extent in the DFT calculations. ${ }^{24}$ That extent inevitably would depend on the DFT approximations to be used. We are concerned with this viewpoint in the present work.

The interlayer binding energy of graphite is also difficult to extract from experiments, and we find only three experiments in the literature. These results are $43 \mathrm{meV} /$ atom from a heat-of-wetting experiment by Girifalco and $\mathrm{Lad}^{3}$ (see also Ref. 24), $35_{-10}^{+15} \mathrm{meV} /$ atom from a microscopic analysis of collapsed carbon nanotubes, ${ }^{39}$ and $52 \pm 5 \mathrm{meV} /$ atom from the experiments on the thermal deposition of polyaromatic hydrocarbons from a graphitic surface. ${ }^{40}$ All these experiments are indirect in obtaining the interlayer binding energy, and the scattered results imply some uncertainties other than those explicitly shown.

In the present work, we revisit the previous semiempirical approach $^{24}$ and explore an improved method of obtaining the interlayer binding energy of graphite. The method is similar to the previous one, but the treatment of the atom-atom vdW interaction is more flexible and covers a wider range of possibility. In this approach, we are also concerned with the development of a useful method that can conveniently be used to study the energetics of other graphitic systems such as carbon nanotubes.

In the next section, we will show for completeness the results of DFT calculations repeated in the standard approximations. In Sec. III, we provide the details of our semiempirical method, which consists of combining those DFT results with an empirical vdW interaction. The results and discussion are given in Sec. IV. The final section is devoted to the conclusions.

\section{DENSITY FUNCTIONAL THEORY CALCULATIONS}

We considered the most stable phase of graphite with $A B A B$ stacking and repeated DFT total-energy calculations in the LDA and GGA approximations using the Vienna $a b$ initio simulation package (VASP) ${ }^{41,42}$ with the projectoraugmented wave method ${ }^{43}$ to describe interaction between electrons and nuclei. We used the exchange-correlation energy functionals of Ceperley and Alder ${ }^{44}$ as parametrized by Perdew and Zunger ${ }^{45}$ for the LDA and of Perdew et al. ${ }^{13}$ for the GGA. The k-space integration was made using the Monkhorst-Pack method ${ }^{46}$ with 56 special $\mathbf{k}$ points in the irreducible Brillouin zone generated from uniform $12 \times 12$ $\times 4$ mesh. The cutoff energy limiting the plane-wave basis set was chosen to be $500 \mathrm{eV}$ in both the LDA and GGA calculations. The present DFT calculations are expected to be more accurate than the previous ones ${ }^{24}$ obtained by using ultrasoft pseudopotential, ${ }^{47}$ the GGA energy functional of Perdew and Wang (PW91),, 12 and the energy cutoff of $358.2 \mathrm{eV}$. The calculated total energy, $E_{\mathrm{DFT}}(a, d)$, as a function of the in-plane lattice constant $a$ and interlayer distance $d=c / 2$ may be written as $E_{\mathrm{DFT}}(a, d)=E_{\mathrm{DFT}}^{0}(a)+U_{\mathrm{DFT}}(a, d)$, where $E_{\mathrm{DFT}}^{0}(a)=E_{\mathrm{DFT}}(a, \infty)$ is the total energy of the isolated graphene sheet and $U_{\mathrm{DFT}}(a, d)$ is the interlayer contribution.
The present DFT calculations for the lattice constants, total cohesive energy $E_{\mathrm{coh}}=-E_{\mathrm{DFT}}(a, d)$, interlayer cohesive energy $U_{\text {coh }}=-U_{\mathrm{DFT}}(a, d)$, and $c$-axis elastic constant $c_{33}$ are summarized and compared to other calculations and experiments in Table I. Here, $U_{\text {coh }}$ is the energy (per atom) required to separate graphite into layers an infinite distance apart. We used the atomic ground-state energy obtained by spinpolarized DFT calculations in extracting $E_{\mathrm{DFT}}(a, d)$ from the calculated total energy, including atomic contribution.

The present results for $a, d$, and $c_{33}$ are in good agreement with the similar calculations by Mounet and Marzari 25 (Table I). These results also agree to varying degrees with earlier calculations, which are summarized in Ref. 24 . The experimental lattice parameters at room temperature obtained by several authors ${ }^{48,50-53}$ are consistent with one another. The in-plane negative thermal expansion at low temperatures ${ }^{25,54}$ implies that the zero-temperature value of $a$ is at most $0.001 \AA$ larger than the room-temperature one and $a$ $\approx 2.462-2.464 \AA$. These values are slightly larger and possibly more accurate than that of Baskin and Meyer ${ }^{48}$ (Table I). However, here and hereafter, we use the latter as the experimental zero-temperature value of $a$ for consistency with the previous calculations. ${ }^{24} \mathrm{We}$ find that such a small difference of $a$ brings about only a marginal influence on the final results. As for the experimental interlayer distance, the zeroand room-temperature values are consistent if we take into account the $c$-axis thermal expansion. ${ }^{54}$ We confirm in Table I that the GGA yields an in-plane lattice constant in an almost complete agreement with experiment but badly fails in predicting interlayer characteristics including $d, U_{\text {coh }}$, and $c_{33}$. In contrast, the agreements between the LDA calculations and experiments for all these interlayer characteristics are much better, with an almost perfect agreement for $d$. We also find that the vdW-DF yields a much better result for $U_{\text {coh }}$ than LDA and GGA, but predicts too large $d$, and there is still room for improvement. The present LDA result for $U_{\text {coh }}$ is qualitatively similar to the previous ones, which are in the range $20-30 \mathrm{meV} / \mathrm{atom},{ }^{16-18,22,24,38}$ with some exceptions. ${ }^{15,19}$ Alfè and Gillan ${ }^{56}$ have reported quantum Monte Carlo calculations of the surface formation energy for the $\mathrm{MgO}$ surface, which also support the accuracy of LDA for this case. These results suggest that the superiority of LDA to GGA is a general feature for the energetics of layered structure and surface, in which sparse electron distributions are involved.

Following the previous work, ${ }^{24}$ we performed DFT calculations for the interlayer interaction energy (per atom), $U_{\mathrm{DFT}}(a, d)$, of graphite in the wide range of $d$ up to $d$ $=7.5 \AA$. In these calculations, the in-plane lattice constant was assumed to be independent of $d$ and set equal to the experimental value, ${ }^{48} a_{\text {expt }}=2.460 \AA$, as we have discussed earlier. Hereafter, the $a$ dependence of $U_{\mathrm{DFT}}(a, d)$ is not explicitly shown unless otherwise stated. For the present purpose, it suffices to know $U_{\mathrm{DFT}}(d)$ as a function of $d$, but it may be useful in applications to other graphitic systems to assume an atom-atom interaction, $\phi_{\mathrm{DFT}}(r)$, giving rise to the calculated $U_{\mathrm{DFT}}(d)$. In this treatment, we employ as before ${ }^{24}$ the continuum model conveniently used in such applications. ${ }^{57}$ Then, the interaction energy between an atom 
TABLE I. Present results of the DFT calculations for the in-plane lattice constants $a$, interlayer spacing $d=c / 2$, total cohesive energy $E_{\mathrm{coh}}$ (in $\mathrm{eV} / \mathrm{atom}$ ), interlayer cohesive energy $U_{\text {coh }}=-U(d)$ (in eV/atom), and $c$-axis elastic constant $c_{33}$ of graphite with $A B A B$ stacking, and their comparisons with other calculations and experiments.

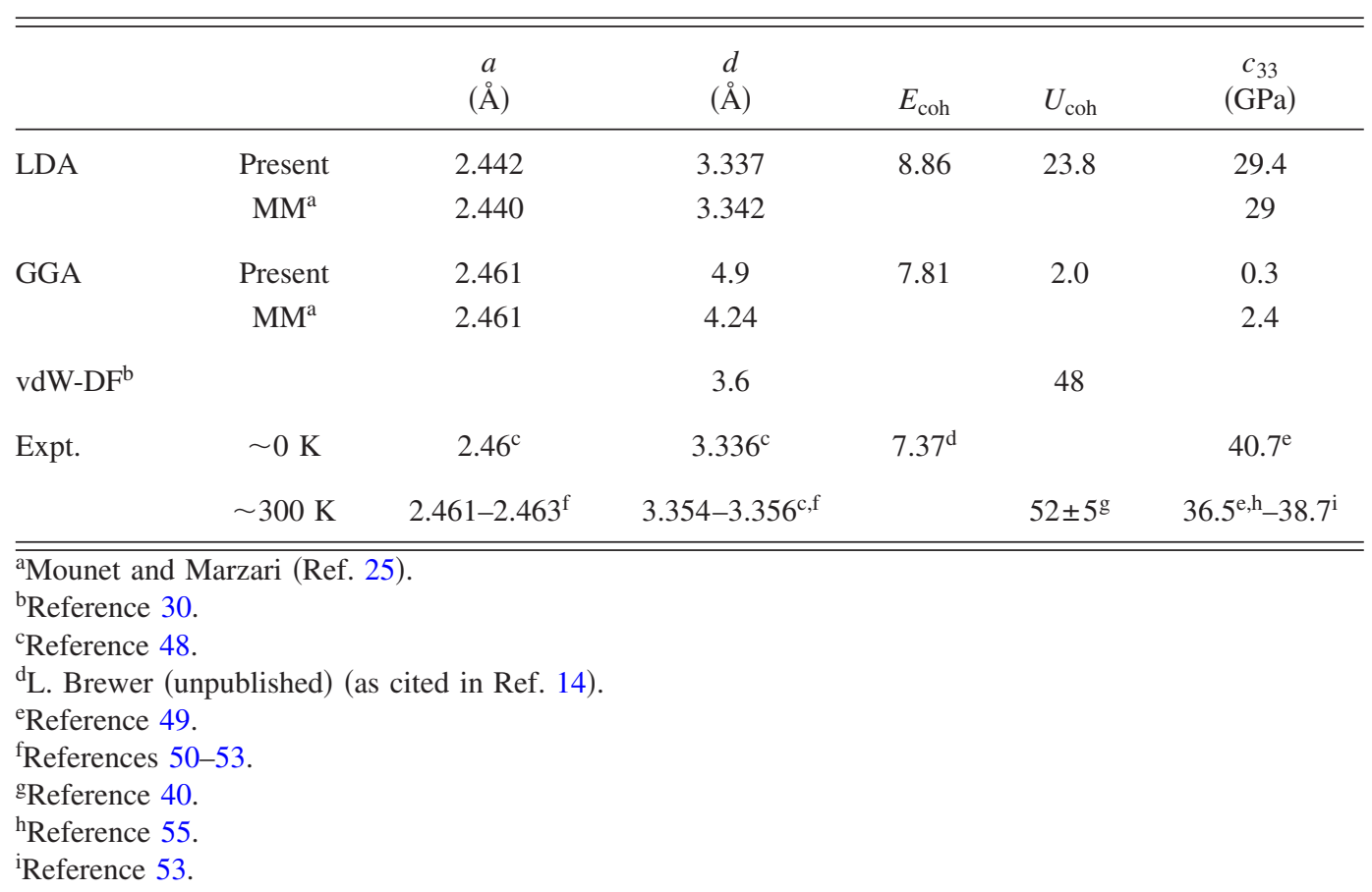

in one layer at $z=0$ and all the atoms in another layer at $z$ $=l d(l= \pm 1, \pm 2, \ldots)$ is given by

$$
\begin{aligned}
V_{\mathrm{DFT}}(l d) & =\int_{0}^{\infty} \phi_{\mathrm{DFT}}\left[\sqrt{(l d)^{2}+r^{2}}\right] 2 \pi \rho r d r \\
& =2 \pi \rho \int_{|l| d}^{\infty} \phi_{\mathrm{DFT}}(r) r d r
\end{aligned}
$$

where $\rho$ is the atomic number density in the layer, $\rho$ $=4 /\left(\sqrt{3} a^{2}\right)$. Using the interlayer interaction energy given by Eq. (1), $U_{\mathrm{DFT}}(d)$ can be written as

$$
U_{\mathrm{DFT}}(d)=\frac{1}{2} \sum_{l=-\infty}^{\infty}{ }^{\prime} V_{\mathrm{DFT}}(l d)=\sum_{l=1}^{\infty} V_{\mathrm{DFT}}(l d),
$$

where the prime on the summation implies the exclusion of the $l=0$ term. We note that $\phi_{\mathrm{DFT}}(r)$ is treated as the interaction between atoms in different layers and cannot be used for describing the atomic interaction within layers. This point is important in some cases as it is in the application to the radial deformation of carbon nanotubes. 57

In such applications to other graphitic systems, it is also useful to parametrize $\phi_{\mathrm{DFT}}(r)$, and we found that it was well represented by a Morse potential, ${ }^{24,57}$

$$
\begin{aligned}
\phi_{\mathrm{DFT}}(r)= & -\frac{M_{0}}{\tau_{2}-\tau_{1}}\left\{\tau_{2} \exp \left[-\tau_{1}(x-1)\right]\right. \\
& \left.-\tau_{1} \exp \left[-\tau_{2}(x-1)\right]\right\},
\end{aligned}
$$

with $x=r / d_{M}$ and $\tau_{2}>\tau_{1}$. We have fitted the four parameters in the above potential to the calculated $U_{\mathrm{DFT}}(d)$ over the range up to $d=7.5 \AA$ by the least-mean-square method and have found that the limiting case, $\tau_{2} \rightarrow \tau_{1}=\tau$, provides the best fit to the LDA results. In this limit, Eq. (3a) reduces to

$$
\phi_{\mathrm{DFT}}(r)=-M_{0}[1+\tau(x-1)] \exp [-\tau(x-1)] .
$$

Both forms of $\phi_{\mathrm{DFT}}(r)$ in Eqs. (3a) and (3b) have the minimum at $r=d_{M}$ with the depth of $M_{0}$. The fitted parameters are summarized in Table II. As we will show later, $U_{\mathrm{DFT}}(d)$ rapidly saturates for large $d$, suggesting that $V_{\mathrm{DFT}}(l d)$ virtually vanishes beyond neighboring separation $(l \geqslant 2)$ even for $d$ as small as $3 \AA$ and $U_{\mathrm{DFT}}(d) \approx V_{\mathrm{DFT}}(d)$. These results clearly indicate that the long-range part of the vdW interaction is not accounted for at all in both the LDA and GGA calculations, although it might have been obvious, from the outset, from their local and semilocal nature.

\section{SEMIEMPIRICAL METHOD}

In our semiempirical method, we assume as before ${ }^{24}$ that the interlayer interaction energy (per atom) is given by the

TABLE II. Parameters of the C-C interaction potentials in Eqs. (3b) and (3a) fitted to the LDA and GGA results, respectively, for the interlayer interaction energy.

\begin{tabular}{lcccc}
\hline \hline & $\begin{array}{c}M_{0} \\
(\mathrm{meV})\end{array}$ & $\begin{array}{c}d_{M} \\
(\AA)\end{array}$ & $\tau\left(=\tau_{1}\right)$ & $\Delta \tau\left(=\tau_{2}-\tau_{1}\right)$ \\
\hline LDA & 2.427 & 3.702 & 10.02 & 0 \\
GGA & 0.054 & 5.167 & 3.453 & 14.73 \\
\hline \hline
\end{tabular}


sum of the DFT result and the additional vdW contribution $U_{\mathrm{vdW}}(d)$ missed in the approximate DFT calculations,

$$
U(d)=U_{\mathrm{DFT}}(d)+U_{\mathrm{vdW}}(d) .
$$

Here, we further assume that $U_{\mathrm{vdW}}(d)$ is given by the sum of the interlayer atom-atom $\mathrm{vdW}$ interaction $\phi_{\mathrm{vdW}}(r)$. Then, again, using the continuum model as in the case of $V_{\mathrm{DFT}}(l d)$ in Eq. (1), the layer-layer vdW interaction can be written as

$$
V_{\mathrm{vdW}}(l d)=2 \pi \rho \int_{|l| d}^{\infty} \phi_{\mathrm{vdW}}(r) r d r .
$$

Using this result, we have

$$
U_{\mathrm{vdW}}(d)=\frac{1}{2} \sum_{l=-\infty}^{\infty}{ }^{\prime} V_{\mathrm{vdW}}(l d)=\sum_{l=1}^{\infty} V_{\mathrm{vdW}}(l d) .
$$

Strictly speaking, the additional contribution cannot necessarily be given in terms of atom-atom interaction, but we may expect, as usual, that such contributions can effectively be incorporated in that form through the empirical determination of $\phi_{\mathrm{vdW}}(r)$, which we describe in the following. This assumption may be accepted as a good approximation for molecular solids including graphite, in which the main body of the interaction is, in fact, given in that form.

The asymptotic form of $\phi_{\mathrm{vdW}}(r)$ obtained by the longrange multipole expansion may be given by $-C_{6} / r^{6}$, but this form is certainly invalid at short distances. The common practice to overcome this deficiency has been to modify the asymptotic form as ${ }^{58}$

$$
\phi_{\mathrm{vdW}}(r)=-\frac{C_{6}}{r^{6}} f_{\mathrm{damp}}(r),
$$

where $f_{\text {damp }}(r)$ is usually called damping function and is chosen to endure the nonsingular behavior of $\phi_{\mathrm{vdW}}(r)$ in the limit $r \rightarrow 0$ and to recover the asymptotic form at large $r$. The damping function in Eq. (7) is usually treated as a universal function independent of atoms or molecules involved. ${ }^{58}$ One of such functions used in the previous studies is of the form $^{33,37}$

$$
f_{\text {damp }}(r) \rightarrow f_{n m}(r)=\left\{1-\exp \left[-\lambda_{0}\left(r / D_{W}\right)^{n}\right]\right\}^{m},
$$

where $D_{W}$ is an appropriately chosen scaling length, and we require $n m \geqslant 6$ to endure the nonsingular behavior of $\phi_{\mathrm{vdW}}(r)$ in the limit $r \rightarrow 0$. As we will see later, we have difficulty in using this type of damping function for our purpose. After several trials, we found that one of the appropriate forms of $f_{\text {damp }}(r)$ is given by

$$
f_{\text {damp }}(r)=\left\{1-\lambda_{2} \exp \left[-\lambda_{1}\left(r / D_{W}\right)^{k}\right]\right\} f_{n m}(r),
$$

which contains four parameters $\left(\lambda_{0}, \lambda_{1}, \lambda_{2}\right.$, and $\left.k\right)$ once $n, m$, and $D_{W}$ are fixed. The short-range behavior of this function is controlled by the prefactor, which is unity, i.e., $f_{\text {damp }}(r)$ $=f_{n m}(r)$, if $\lambda_{2}=0$ and changes sign at some separation if $\lambda_{2}$ $>1$, as actually happens to the LDA-based method. In this way, the form of $f_{\text {damp }}(r)$ in Eq. (9) covers the ordinary ones with a monotonic behavior and is more flexible with a wider range of possibility. A more explicit motivation to assume that form will be discussed later.
Using Eq. (7) in Eq. (5), $V_{\mathrm{vdw}}(l d)(l>0)$ can be calculated as

$$
\begin{aligned}
V_{\mathrm{vdW}}(l d) & =-2 \pi \rho C_{6} \int_{l d}^{\infty} \frac{1}{r^{5}} f_{\mathrm{damp}}(r) d r \\
& =-2 \pi \rho C_{6}\left\{\int_{l d}^{\infty} \frac{1}{r^{5}} d r-\int_{l d}^{\infty} \frac{1}{r^{5}}\left[1-f_{\mathrm{damp}}(r)\right] d r\right\} \\
& =-\frac{\pi \rho C_{6}}{2 d^{4}}\left\{1 / l^{4}-4 J_{l}(d)\right\},
\end{aligned}
$$

where

$$
J_{l}(d)=\int_{l}^{\infty}\left[1-f_{\mathrm{damp}}(y d)\right] y^{-5} d y .
$$

Then, using Eq. (10) in Eq. (6), we have

$$
U_{\mathrm{vdW}}(d)=-\frac{\pi \rho C_{6}}{2 d^{4}}\left\{\zeta(4)-4 \sum_{l=1}^{l_{\max }} J_{l}(d)\right\},
$$

where $\zeta(4)=\pi^{4} / 90, \zeta(n)$ being Riemann's zeta function defined by $\zeta(n)=1+1 / 2^{n}+1 / 3^{n}+\cdots$, and $J_{l}(d)$ virtually vanishes for $l>l_{\max }=3-4$.

The parameters of $f_{\text {damp }}(r)$ in Eq. (9) are determined by requiring the following physical and theoretically motivated conditions. (i) First, the interlayer interaction energy $U(d)$, given by Eq. (4), should be consistent with the experimental interlayer spacing at $T=0 \mathrm{~K}$, i.e., $U^{\prime}\left(d_{\text {expt }}\right)=U_{\mathrm{DFT}}^{\prime}\left(d_{\text {expt }}\right)$ $+U_{\text {vdw }}^{\prime}\left(d_{\text {expt }}\right)=0$, with $d_{\text {expt }}=3.336 \AA^{48}$ (ii) Similarly, the $c$-axis elastic constant at $T=0 \mathrm{~K}$ is reproduced, i.e., $c_{33}$ $=\rho d_{\text {expt }} U^{\prime \prime}\left(d_{\text {expt }}\right)=40.7 \mathrm{GPA}^{49}$ (iii) The experimental inplane lattice constant, $a_{\text {expt }}=2.460 \AA{ }^{48}$ is also reproduced, i.e., $\partial E\left(a, d_{\text {expt }}\right) / \partial a=0$ at $a=a_{\text {expt }}$. Here, $E(a, d)$ is the total energy of graphite and is given by $E(a, d)=E_{\mathrm{DFT}}(a, d)$ $+E_{\mathrm{vdw}}(a, d)$, with $E_{\mathrm{DFT}}(a, d)$ and $E_{\mathrm{vdw}}(a, d)$ being the result of DFT calculation and the supplemented vdW contribution, respectively. $E_{\mathrm{vdw}}(a, d)$ is given by the sum of $U_{\mathrm{vdw}}(d)$ and the in-plane vdW contribution, $E_{\mathrm{vdw}}^{0}(a)=E_{\mathrm{vdw}}(a, d \rightarrow \infty)$. We may assume that the interlayer and in-plane atom-atom vdW interactions are the same. Then, again, using the continuum model to be consistent with the calculation of $U_{\mathrm{vdw}}(d)$, the in-plane vdW contribution can easily be calculated as

$$
\begin{aligned}
E_{\mathrm{vdW}}^{0}(a) & =\frac{1}{2} \int_{0}^{\infty} \phi_{\mathrm{vdW}}(r) 2 \pi \rho r d r \\
& =-\pi \rho C_{6}\left\{\int_{0}^{R_{\max }} \frac{1}{r^{5}} f_{\mathrm{damp}}(r) d r+\frac{1}{4 R_{\max }^{4}}\right\},
\end{aligned}
$$

where $R_{\max } \approx l_{\max } d$ and $f_{\text {damp }}(r)$ virtually vanishes for $r$ $>R_{\max }$ as before. (iv) The final requirement is that the LDAand GGA-based methods (LDA+vdW, GGA+vdW) yield the same $U\left(d_{\text {expt }}\right)$ with the assumption that $f_{n m}(r)$ in Eq. (9) is a universal function independent of DFT approximation. At this point, we note again that the vdW term, $U_{\mathrm{vdw}}(d)$, is assumed to be the correction to the approximate DFT calcu- 
lations. Hence, if such a correction is properly made, the combined result, $U(d)$, should be the same irrespective of the underlying DFT approximation. The final requirement is nothing but the consequence of this theoretically motivated necessary condition.

Here, we come back to the damping function given by Eq. (9) and clarify the practical motivation leading us to that form. From Eqs. (4), (6), and (10), we have

$$
U^{\prime}(d)=U_{\mathrm{DFT}}^{\prime}(d)+\frac{2 \pi \rho C_{6}}{d^{5}} \sum_{l=1}^{\infty} \frac{1}{l^{4}} f_{\mathrm{damp}}(l d) .
$$

If we use the LDA result $(\mathrm{DFT} \rightarrow \mathrm{LDA}), U_{\mathrm{LDA}}^{\prime}\left(d_{\text {expt }}\right) \approx 0$ since LDA almost perfectly predicts the interlayer spacing (see Table I), implying that the second term on the right-hand side of Eq. (14) must also vanish in order that $U(d)$ satisfies the first requirement, $U^{\prime}\left(d_{\text {expt }}\right)=0$. More specifically, $f_{\text {damp }}\left(d_{\text {expt }}\right)$ must be negative since $f_{\text {damp }}\left(l d_{\text {expt }}\right)$ with $l>1$ would be positive and nearly equal to unity. The ordinary damping functions, such as that in Eq. (8), certainly show no such behavior. This difficulty of the LDA-based route $(\mathrm{LDA}+\mathrm{vdW})$ was the reason why we took a different approach in the previous work. ${ }^{24}$ In that approach the layerlayer interaction energies, $V_{\mathrm{DFT}}(l d)$ and $V_{\mathrm{vdw}}(l d)$ [with $\left.f_{\text {damp }}(l d)=1\right]$, were interpolated using a Fermi function with two parameters, which were determined by the first and second requirements discussed earlier. This interpolation scheme was similar to that used by Pacheco and Ramalho ${ }^{59}$ and Hasegawa et al. ${ }^{60}$ in their calculations of the intermolecular potential in $\mathrm{C}_{60}$ solids. We have no such difficulty in using the GGA calculations, and this is probably the reason why Ortmann et al. ${ }^{37,38}$ did not use the LDA result in their semiempirical approach but concentrated only on the GGAbased method (i.e., GGA $+v d W$ ). The modification of $f_{\text {damp }}(r)$ in Eq. (9) was motivated by this practical difficulty, thereby enabling us to make a systematic, consistent treatment of the LDA and GGA calculations.

\section{RESULTS AND DISCUSSION}

We used the vdW constant, $C_{6}=16.34 \mathrm{eV} \AA^{6}$, obtained by $\mathrm{Wu}$ and Yang $^{33}$ in their analyses of the interatomic vdW interactions as an appropriate value for the carbon atoms in the $s p^{2}$ bonding state. We also employed $f_{32}(r)$ used by them $(n=3$ and $m=2)$ for $f_{n m}(r)$ in Eq. (9), in which the sum of the atomic vdW radii is used as the scaling length, i.e., $D_{W}$ $=2 R_{W}$ with $R_{W}=1.70 \AA$ for carbon atoms ${ }^{61}$ With this choice of the scaling length, the present damping function $f_{\text {damp }}(r)$

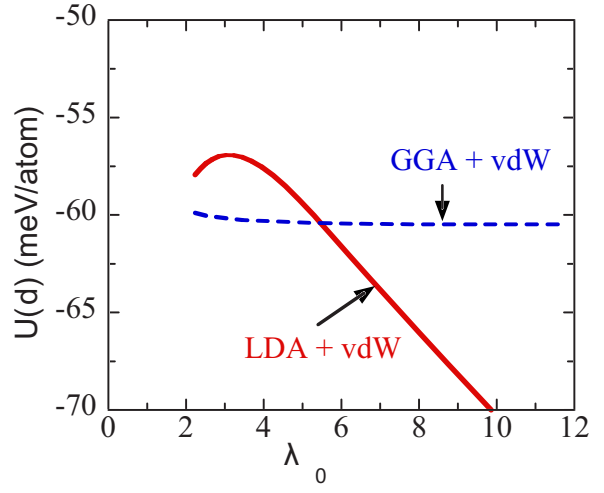

FIG. 1. (Color online) Interlayer interaction energies $U(d)$ at the experimental interlayer spacing, $d=d_{\text {expt }}$, as functions of $\lambda_{0}$ [the parameter of $\left.f_{32}(r)\right]$. For each $\lambda_{0}, U(d)$ was determined by the physical conditions, (i)-(iii), described in the text. Both results for $U(d)(\mathrm{LDA}+\mathrm{vdW}$ and GGA $+\mathrm{vdW})$ coincide with each other at $\lambda_{0}=5.467$, thereby satisfying the theoretically motivated requirement (iv), and is equal to $-60.4 \mathrm{meV} / \mathrm{atom}$.

given by Eq. (9) is expected to be universal; i.e., its parameters $\left(\lambda_{0}, \lambda_{1}, \lambda_{2}\right.$, and $\left.k\right)$ are independent of atoms involved. However, it is difficult to confirm this universality at this stage because it requires detailed experimental data for other systems. The procedure to determine the parameters of $f_{\text {damp }}(r)$ is as follows. For a given value of $\lambda_{0}$ in $f_{32}(r)$, the remaining parameters of $f_{\text {damp }}(r)$ are determined by the physical requirements, (i)-(iii), described in the previous section. Figure 1 shows the resulting interlayer interaction energy $U\left(d_{\text {expt }}\right)$ as functions of $\lambda_{0}$ obtained in this way. We find that the LDA-based result $(\mathrm{LDA}+\mathrm{vdW})$ for $U\left(d_{\text {expt }}\right)$ shows a large variation with $\lambda_{0}$, while the GGA-based result $(\mathrm{GGA}+\mathrm{vdW})$ is almost constant (slightly decreasing with $\left.\lambda_{0}\right)$. Both results coincide with each other at $\lambda_{0}=5.467$, thereby satisfying the theoretically motivated final requirement. This value of $\lambda_{0}$ is in between those originally used by Mooij et al. ${ }^{62}\left(\lambda_{0}=7.19\right)$ to simulate the damping function of Ahlrichs et al..$^{63}$ and later corrected by $\mathrm{Wu}$ and Yang ${ }^{33}$ $\left(\lambda_{0}=3.54\right)$, which simulates more likely that function. However, these damping functions cannot directly be compared to the present $f_{32}(r)$, which is treated as the intrinsic part of the damping function and assumed to be independent of the DFT approximation.

The parameters of $f_{\text {damp }}(r)$ for the above value of $\lambda_{0}$ are summarized in Table III, and the corresponding results for $U(d)$ as functions of $d$ are illustrated in Fig. 2. The LDA- and GGA-based results (LDA+vdW, GGA+vdW) for $U(d)$, $U^{\prime}(d)$, and $U^{\prime \prime}(d)$ are the same at $d=d_{\text {expt }}$ by construction.

TABLE III. Parameters of the damping function in Eq. (9), with the Wu-Yang type of $f_{n m}(r)(n=3, m$ $=2$ ), determined by imposing the conditions described in the text. Also included are the in-plane and interlayer vdW contributions ( $E_{\mathrm{vdW}}^{0}$ and $U_{\mathrm{vdW}}$, respectively, in units of eV/atom) to the total energy of graphite at the experimental lattice constants.

\begin{tabular}{lcccccc}
\hline \hline & $\lambda_{0}$ & $\lambda_{1}$ & $\lambda_{2}$ & $\kappa$ & $E_{\mathrm{vdW}}^{0}$ & $U_{\mathrm{vdW}}$ \\
\hline LDA+vdW & 5.467 & 0.591 & 1.882 & 3.315 & 0.511 & -0.037 \\
GGA + vdW & 5.467 & 0.909 & 0.983 & 7.139 & -0.071 & -0.075 \\
\hline \hline
\end{tabular}




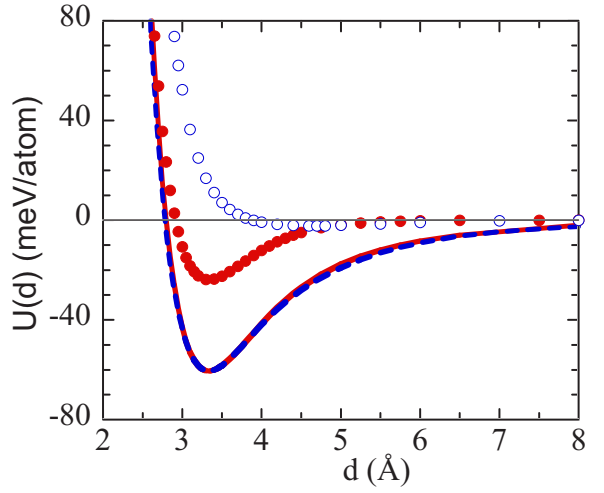

FIG. 2. (Color online) Interlayer interaction energies as functions of the interlayer spacing obtained by the LDA (closed circles) and GGA (open circles) calculations and by the semiempirical methods, LDA+vdW (solid line) and GGA+vdW (dashed line), both being almost indistinguishable from each other.

Both routes also yield quite similar results over the wide range of $d$, which is the favorable feature coming out without any additional requirement and enforces our confidence in the validity as well as the usefulness of the present semiempirical approach. The interlayer cohesive energy $U_{\text {coh }}=-U\left(d_{\text {expt }}\right)$ obtained in this way is $60.4 \mathrm{meV} /$ atom, which is close to the previous semiempirical results ${ }^{24}$ (60.7 meV/atom for LDA+vdW and $57.4 \mathrm{meV} / \mathrm{atom}$ for $\mathrm{GGA}+\mathrm{vdW})$ and somewhat larger than the empirical one obtained with the use of LJ potential (55.5 meV/atom). We note that in our previous semiempirical method, ${ }^{24}$ we have not required that the two routes ( $\mathrm{LDA}+\mathrm{vdW}$ and GGA $+\mathrm{vdW})$ should yield the same $U\left(d_{\text {expt }}\right)$. Ortmann et al. ${ }^{38} \mathrm{ob}-$ tained a somewhat larger value of $U_{\text {coh }}=80.2 \mathrm{meV} /$ atom by their method $(\mathrm{GGA}+\mathrm{vdW})$ using $f_{81}(r)(n=8, m=1)$ as the damping function. They determined its parameter for graphite only by the first requirement, $U^{\prime}\left(d_{\text {expt }}\right)=0$, and treated $f_{81}(r)$, as usual, as a universal function applicable to any system. Their result suggests that such a simple treatment of the damping function overestimates $U_{\mathrm{coh}}$. At room temperature, $U_{\text {coh }}$ is lowered by $\sim 6.5 \mathrm{meV} /$ atom by the thermal effect compared to that at $T=0 \mathrm{~K}$ and reduces to

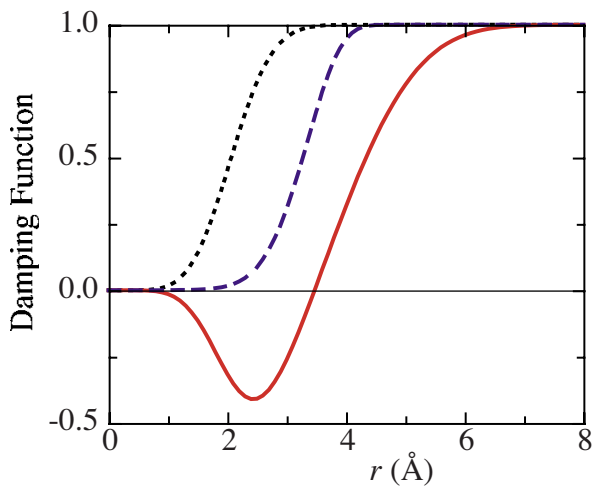

FIG. 3. (Color online) Damping functions $f_{\text {damp }}(r)$ given by Eq. (9) (with the parameters in Table III) in the LDA+vdW (solid line) and GGA+vdW (dashed line) methods. Also shown is the intrinsic part, $f_{32}(r)$ (dotted line).
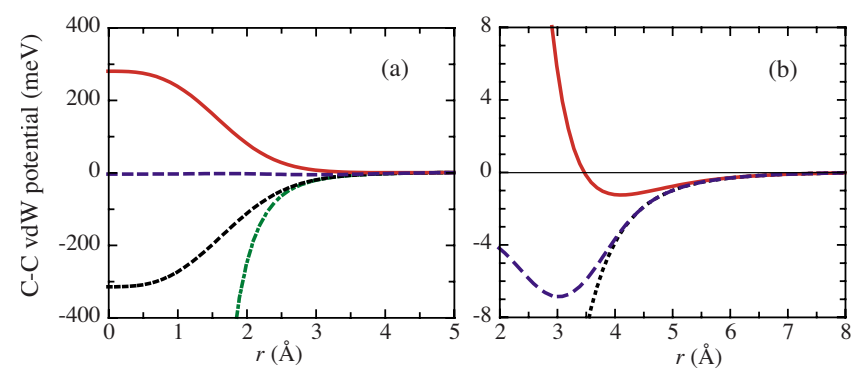

FIG. 4. (Color online) C-C vdW interaction potentials $\phi_{\mathrm{vdW}}(r)$ given by Eq. (7) in the $\mathrm{LDA}+\mathrm{vdW}$ (solid line) and GGA+vdW (dashed line) methods. Also shown are $-\left(C_{6} / r^{6}\right) f_{32}(r)$ (dotted line) and the asymptotic form $-C_{6} / r^{6}$ (dash-dot line). In (b), the vertical scale is expanded 50 times.

$\sim 54 \mathrm{meV} /$ atom. ${ }^{24}$ This result is consistent with the most recent experiment by Zacharia et al., ${ }^{40} 52 \pm 5 \mathrm{meV} /$ atom, although uncertainties in experimental results are unavoidable, as we have discussed earlier.

The damping functions with the parameters summarized in Table III are illustrated in Fig. 3. As we have anticipated beforehand, $f_{\text {damp }}(r)$ for the LDA-based method (LDA $+\mathrm{vdW})$ is negative in the short range, $r<3.5 \AA$, and, as a consequence, $\phi_{\mathrm{vdW}}(r)$ is large and positive in that range, as illustrated in Fig. 4, quite in contrast to the ordinary ones. On the other hand, the GGA-based route $(\mathrm{GGA}+\mathrm{vdW})$ yields the results of $f_{\text {damp }}(r)$ and $\phi_{\mathrm{vdW}}(r)$ similar to the ordinary ones, although their magnitudes in the short range are much reduced. As we have already discussed, the short-range part of the present $\phi_{\mathrm{vdW}}(r)$ is interpreted as containing, in addition to the ordinary vdW interaction, the contribution correcting for the approximate DFT calculations and inevitably depends on the underlying DFT approximation as actually visualized for the LDA and GGA. Figure 5 illustrates the C-C interactions $\phi_{\mathrm{DFT}}(r)$ given by Eq. (3a) or (3b) fitted to the LDA and GGA results for $U_{\mathrm{DFT}}(d)$ [see Eq. (2)] and the

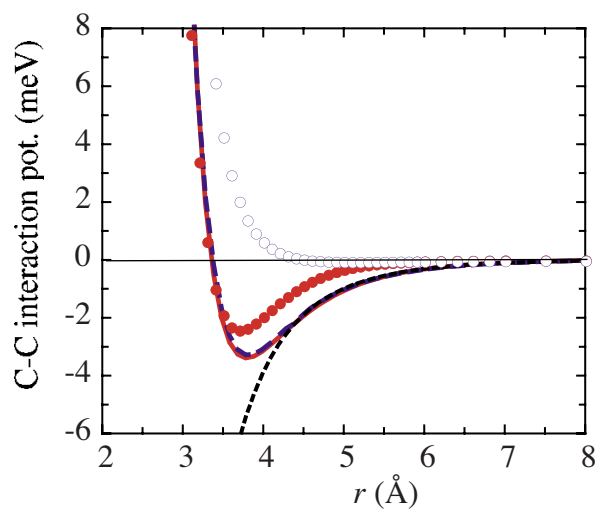

FIG. 5. (Color online) Interlayer C-C interaction potentials $\phi_{\mathrm{DFT}}(r)$ fitted to the calculated $U_{\mathrm{DFT}}(d)$ in the LDA (closed circles) and GGA (open circles) and the total potential, $\phi(r)=\phi_{\mathrm{DFT}}(r)$ $+\phi_{\mathrm{vdW}}(r)$, obtained by the LDA $+\mathrm{vdW}$ (solid line) and GGA $+\mathrm{vdW}$ (dashed line) methods, both being almost indistinguishable from each other. Also shown is the asymptotic form $-C_{6} / r^{6}$ (dotted line). 
corresponding total C-C interactions, $\quad \phi(r)=\phi_{\mathrm{DFT}}(r)$ $+\phi_{\mathrm{vdW}}(r)$. These results provide a useful means to investigate the energetics of the radial deformation of carbon nanotubes ${ }^{57}$ where $\phi_{\mathrm{DFT}}(r)$ and $\phi_{\mathrm{vdW}}(r)$ are conveniently treated separately. These results for $\phi(r)$ may also be directly applicable to the calculations of the wall-wall interaction in multiwall carbon nanotubes and the interaction between nanotubes in bundles.

The short-range part of $\phi_{\mathrm{vdW}}(r)(r<d)$ has no direct influence on the interlayer interaction itself but has a substantial effect on the in-plane energetics in the case of LDA. In fact, with the inclusion of the vdW interaction, the LDA total cohesive energy (see Table I), $E_{\text {coh }}$, at experimental lattice constants lowers by $0.47 \mathrm{eV} /$ atom with 0.51 and $-0.04 \mathrm{eV} /$ atom as the in-plane and interlayer contributions, respectively (see Table III) and reduces to $E_{\mathrm{coh}}=8.39 \mathrm{eV} /$ atom. On the other hand, $E_{\mathrm{coh}}$ in the GGA is increased by $0.146 \mathrm{eV} /$ atom with comparable in-plane and interlayer contributions $(0.071$ and $0.075 \mathrm{eV} /$ atom, respectively), and we have $E_{\text {coh }}=7.96 \mathrm{eV} /$ atom. These results indicate that the supplemented vdW interactions favorably correct for the overbinding and underbinding nature of LDA and GGA, respectively, thereby providing further confidence in the present method characterized by the damping function in Eq. (9). The corrected results of $E_{\text {coh }}$ in the LDA and GGA are still different by $\sim 0.43 \mathrm{eV} /$ atom. This difference may partly be ascribed to the so-called multiconfiguration correction in the atomic ground-state energy calculations, which amounts to $\sim 1.4 \mathrm{eV}$ in the Hartree-Fock calculations ${ }^{64}$ and could be substantially different between LDA and GGA.

All these results were obtained for the damping function $f_{\text {damp }}(r)$, with $f_{32}(r)$ as the intrinsic part. We also tried $f_{42}(r)$ and $f_{81}(r)$ in place of $f_{32}(r)$, with $f_{81}(r)$ being the damping function used by Ortmann et al. ${ }^{37,38}$ and found that the results for $U(d)$ are almost indistinguishable from those obtained by using $f_{32}(r)$. The vdW interactions $\phi_{\mathrm{vdW}}(r)$ corresponding to $f_{42}(r)$ and $f_{81}(r)$ vanish at $r=0$ and are different from that for $f_{32}(r)$ in the short range, but this difference is minimal in the physically relevant range, $r>r_{\mathrm{CC}}=1.42 \AA$, where $r_{\mathrm{CC}}$ is the $\mathrm{C}-\mathrm{C}$ bond length in the layer. We also note that the continuum model used in the present approach makes the implementation of our semiempirical method quite simple without sacrificing quantitative accuracy. This model is much more useful in applications of the present results to other graphitic systems, ${ }^{57}$ for which the discreteatom model brings about formidable complexities in implementations with little quantitative advantage.

\section{CONCLUSIONS}

We have revisited the previous semiempirical approach ${ }^{24}$ and developed an improved method of calculating the interlayer binding energy of graphite. This method is motivated by the basic idea that the supplemented atom-atom interaction, typically vdW interaction, should contain all the contributions that approximate DFT calculations fail to take into account. In accordance with this viewpoint, we introduced a flexible damping function for the atom-atom vdW interaction, which covers a wider range of possibility and inevitably depends on the underlying DFT approximations. We also successfully aimed at a consistent treatment of the supplemented vdW interaction in the LDA- and GGA-based method and obtained $U_{\text {coh }}=60.4 \mathrm{meV} /$ atom as the interlayer cohesive energy at $T=0 \mathrm{~K}$. This result is, if corrected by the thermal effect, consistent with the most resent experiment. ${ }^{40}$ The present semiempirical result for $U_{\text {coh }}$ may be the most plausible among others owing to the imposed necessary conditions, although it is not much different from the previous ones obtained by the empirical, ${ }^{3-5,24}$ semiempirical, ${ }^{24}$ and $\mathrm{vdW}-\mathrm{DF}^{30}$ methods. The $\mathrm{C}-\mathrm{C} \mathrm{vdW}$ interaction $\phi_{\mathrm{vdW}}(r)$ involved in the present method differs from the previous ones and provides a useful insight into the failure and limitation of the standard DFT approximations for systems with sparse electron distribution. We actually confirmed that the supplemented vdW interaction favorably corrects for the overbinding and underbinding nature of LDA and GGA, respectively, in the in-plane energetics of graphite. Besides our original scope for the interlayer binding in graphite, the present method may also provide a useful starting point for studying the energetics of other graphitic systems. In fact, using the atom-atom interactions, $\phi_{\mathrm{DFT}}(r)$ and $\phi_{\mathrm{vdW}}(r)$, for graphite obtained by our method, we have already developed a simple theory for the radial deformation of single-walled carbon nanotubes. ${ }^{57}$ These results can also be directly applicable to the calculations of the wall-wall interaction in multiwalled carbon nanotubes and the interaction between nanotubes in bundles.

\section{ACKNOWLEDGMENTS}

We gratefully acknowledge the support by the Grant-inAid for Scientific Research from JSPS under Grant No. 16540301. The computations in this work were performed using the computer facilities of the Information Processing Center, Iwate University and of the Institute for Solid State Physics, University of Tokyo.

\footnotetext{
*hasegawa@iwate-u.ac.jp

${ }^{1}$ See, for example, M. S. Dresselhaus, G. Dresselhaus, and P. C. Eklund, Science of Fullerenes and Carbon Nanotubes (Academic, San Diego, 1996).

${ }^{2}$ L. A. Girifalco and M. Hodak, Phys. Rev. B 65, 125404 (2002), and references therein.

${ }^{3}$ L. A. Girifalco and R. A. Lad, J. Chem. Phys. 25, 693 (1956).
}

${ }^{4}$ L. A. Girifalco, M. Hodak, and R. S. Lee, Phys. Rev. B 62, 13104 (2000).

${ }^{5}$ H. Ulbricht, G. Moos, and T. Hertel, Phys. Rev. Lett. 90, 095501 (2003).

${ }^{6}$ P. Hohenberg and W. Kohn, Phys. Rev. 136, B864 (1964).

${ }^{7}$ W. Kohn and L. Sham, Phys. Rev. 140, A1133 (1965).

${ }^{8}$ D. C. Langreth and M. J. Mehl, Phys. Rev. B 28, 1809 (1983). 
${ }^{9}$ J. P. Perdew and Y. Wang, Phys. Rev. B 33, 8800 (1986); 40, 3399(E) (1989).

${ }^{10}$ J. P. Perdew, Phys. Rev. B 33, 8822 (1986); 34, 7406(E) (1986).

${ }^{11}$ A. D. Becke, Phys. Rev. A 38, 3098 (1988).

${ }^{12}$ J. P. Perdew, in Electronic Structure of Solids '91, edited by P. Ziesche and H. Eschrig (Akademie-Verlag, Berlin, 1991), p. 11.

${ }^{13}$ J. P. Perdew, K. Burke, and M. Ernzerhof, Phys. Rev. Lett. 77, 3865 (1996).

${ }^{14}$ M. T. Yin and M. L. Cohen, Phys. Rev. B 29, 6996 (1984).

${ }^{15}$ H. J. F. Jansen and A. J. Freeman, Phys. Rev. B 35, 8207 (1987).

${ }^{16}$ S. B. Trickey, F. Müller-Plathe, G. H. F. Diercksen, and J. C. Boettger, Phys. Rev. B 45, 4460 (1992).

${ }^{17}$ M. C. Schabel and J. L. Martins, Phys. Rev. B 46, 7185 (1992).

${ }^{18}$ J.-C. Charlier, X. Gonze, and J.-P. Michenaud, Europhys. Lett. 28, 403 (1994).

${ }^{19}$ B. I. Dunlap and J. C. Boettger, J. Phys. B 29, 4907 (1996).

${ }^{20}$ J. C. Boettger, Phys. Rev. B 55, 11202 (1997).

${ }^{21}$ G. Kern and J. Hafner, Phys. Rev. B 58, 13167 (1998).

${ }^{22}$ Y. C. Wang, K. Scheerschmidt, and U. Gösele, Phys. Rev. B 61, 12864 (2000).

${ }^{23}$ G. Sun, M. Kertesz, J. Kürti, and R. H. Baughman, Phys. Rev. B 68, 125411 (2003).

${ }^{24}$ M. Hasegawa and K. Nishidate, Phys. Rev. B 70, 205431 (2004).

${ }^{25}$ N. Mounet and N. Marzari, Phys. Rev. B 71, 205214 (2005).

${ }^{26}$ H. Rydberg, B. I. Lundqvist, D. C. Langreth, and M. Dion, Phys. Rev. B 62, 6997 (2000).

${ }^{27}$ H. Rydberg, M. Dion, N. Jacobson, E. Schröder, P. Hyldgaard, S. I. Simak, D. C. Langreth, and B. I. Lundqvist, Phys. Rev. Lett. 91, 126402 (2003).

${ }^{28}$ H. Rydberg, N. Jacobson, P. Hyldgaard, S. I. Simak, B. I. Lundqvist, and D. C. Langreth, Surf. Sci. 532-535, 606 (2003).

${ }^{29}$ M. Dion, H. Rydberg, E. Schröder, D. C. Langreth, and B. I. Lundqvist, Phys. Rev. Lett. 92, 246401 (2004); 95, 109902(E) (2005).

${ }^{30}$ S. D. Chakarova-Käck, E. Schröder, B. I. Lundqvist, and D. C. Langreth, Phys. Rev. Lett. 96, 146107 (2006).

${ }^{31}$ M. Elstner, P. Hobza, T. Fraunheim, and Kaxiras, J. Chem. Phys. 114, 5149 (2001).

${ }^{32}$ X. Wu, M. C. Vargas, S. Nayak, V. Lotrich, and G. Scoles, J. Chem. Phys. 115, 8748 (2001).

${ }^{33}$ Q. Wu and W. Yang, J. Chem. Phys. 16, 515 (2002).

${ }^{34}$ U. Zimmerli, M. Parrinello, and P. Koumoutsakos, J. Chem. Phys. 120, 2693 (2004).

${ }^{35}$ S. Grimme, J. Comput. Chem. 25, 1463 (2004).

${ }^{36}$ M. A. Neumann and M.-A. Perrin, J. Phys. Chem. B 109, 15531 (2005).
${ }^{37}$ F. Ortmann, W. G. Schmidt, and F. Bechstedt, Phys. Rev. Lett. 95, 186101 (2005).

${ }^{38}$ F. Ortmann, F. Bechstedt, and W. G. Schmidt, Phys. Rev. B 73, 205101 (2006).

${ }^{39}$ L. X. Benedict, N. G. Chopra, M. L. Cohen, A. Zettl, S. G. Louie, and V. H. Crespi, Chem. Phys. Lett. 286, 490 (1998).

${ }^{40}$ R. Zacharia, H. Ulbricht, and T. Hertel, Phys. Rev. B 69, 155406 (2004).

${ }^{41}$ G. Kresse and J. Hafner, Phys. Rev. B 47, R558 (1993); 49, 14251 (1994).

${ }^{42}$ G. Kresse and J. Furthmüller, Phys. Rev. B 54, 11169 (1996); Comput. Mater. Sci. 6, 15 (1996).

${ }^{43}$ G. Kresse and D. Joubert, Phys. Rev. B 59, 1758 (1999).

${ }^{44}$ D. M. Ceperley and B. J. Alder, Phys. Rev. Lett. 45, 566 (1980).

${ }^{45}$ J. P. Perdew and A. Zunger, Phys. Rev. B 23, 5048 (1981).

${ }^{46}$ H. J. Monkhorst and J. D. Pack, Phys. Rev. B 13, 5188 (1976).

${ }^{47}$ D. Vanderbilt, Phys. Rev. B 41, 7892 (1990).

${ }^{48}$ Y. Baskin and L. Meyer, Phys. Rev. 15, 544 (1955).

${ }^{49}$ W. B. Gauster and I. J. Fritz, J. Appl. Phys. 45, 3309 (1974).

${ }^{50} \mathrm{P}$. Trucano and R. Chen, Nature (London) 258, 136 (1975).

${ }^{51}$ M. Hanfland, H. Beister, and K. Syassen, Phys. Rev. B 39, 12598 (1989).

${ }^{52}$ Y. X. Zhao and I. L. Spain, Phys. Rev. B 40, 993 (1989).

${ }^{53}$ A. Bosak, M. Krisch, M. Mohr, J. Maultzsch, and C. Thomsen, Phys. Rev. B 75, 153408 (2007).

${ }^{54}$ A. C. Baily and B. Yates, J. Appl. Phys. 41, 5088 (1970).

${ }^{55}$ O. L. Blakslee, D. G. Proctor, E. J. Seldin, G. B. Spence, and T. Weng, J. Appl. Phys. 41, 3373 (1970).

${ }^{56}$ D. Alfè and M. J. Gillan, J. Phys.: Condens. Matter 18, L435 (2006).

${ }^{57}$ M. Hasegawa and K. Nishidate, Phys. Rev. B 74, 115401 (2006).

${ }^{58}$ A. J. Stone, The Theory of Intermolecular Forces (Oxford University Press, New York, 1996).

${ }^{59}$ J. M. Pacheco and J. P. Prates Ramalho, Phys. Rev. Lett. 79, 3873 (1997).

${ }^{60}$ M. Hasegawa, K. Nishidate, M. Katayama, and T. Inaoka, J. Chem. Phys. 119, 1386 (2003).

${ }^{61}$ A. Bondi, J. Phys. Chem. 68, 441 (1964).

${ }^{62}$ W. T. M. Mooij, F. B. van Duijneveldt, J. G. C. M. van Duijneveldt-van de Rijdt, and B. P. van Eijck, J. Phys. Chem. A 103, 9872 (1999).

${ }^{63}$ R. Ahlrichs, R. Penco, and G. Scoles, Chem. Phys. 19, 119 (1977).

${ }^{64}$ M. Weinert, E. Wimmer, and A. J. Freeman, Phys. Rev. B 26, 4571 (1982). 